\title{
Detector Depth Dependence of the High Energy Atmospheric Neutrino Flux
}

\author{
John M. LoSecct* \\ Physics Department, University of Notre Dame, Notre Dame, Indiana 46556 USA
}

(Dated: November 20, 2018)

\begin{abstract}
We note that detector depth can influence the decay path length available for the primary and secondary particles that are the source of atmospheric neutrinos. As a consequence there is a location dependent modulation to the neutrino flux, which could be as large as $5-10 \%$ in some directions.
\end{abstract}

\section{INTRODUCTION}

Estimates for the ambient neutrino flux are an important input parameter to experiments analyzing underground neutrino interactions and upward going muons to understand the time evolution of a neutrino beam. Different directions provide neutrino fluxes with varying source distance so observation of the angular distribution is an essential tool in the study of neutrino oscillations. Since the overall flux normalization is uncertain, experiments frequently place a greater emphasis on the shape of the distribution than the absolute event rate.

This note points out depth dependent effects that can also provide a directional modulation. These effects are modest, but predominantly effect the higher end of the neutrino spectrum.

Upward going muons [1, 2, 3, 4, 5] are usually attributed to muon neutrino interactions in the rock surrounding the detector. In order to be detected as an upward going muon, the lepton produced in a neutrino interaction must propagate through the rock to be recorded in the detector. If we approximate the muon energy loss as muon energy independent then the range increases linearly with the muon energy. So the target rock surrounding the detector has a larger effective mass for neutrino interactions at high energy, scaling roughly as $E_{\nu}^{2}$. Over a substantial range of neutrino energies the cross section rises linearly. So that a constant mass detector will have more high energy neutrino interactions than low energy neutrino interactions at the same flux. These two factors suggest that the neutrino induced muon flux is sensitive to the third power of the neutrino energy. Small neutrino flux differences at high energies are substantially amplified by this $E_{\nu}^{3}$ factor.

We present a one dimensional model to show that the atmospheric decay path length is a function of the detector depth. Detectors which are above sea level will see neutrinos with a higher decay path length than detectors below sea level. To first order the high energy part of the neutrino flux is proportional to the decay path length.

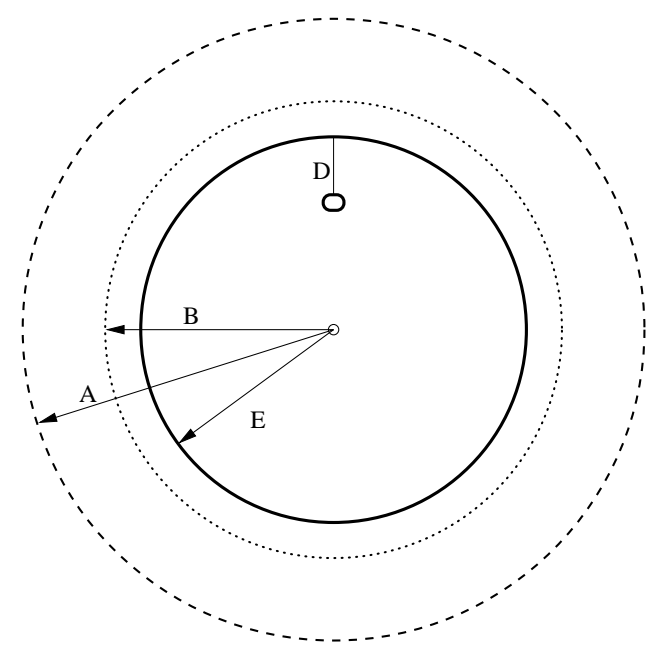

FIG. 1: The atmospheric neutrino source geometry. This figure is not to scale

*Electronic address: losecco@undhep.hep.nd.edu 


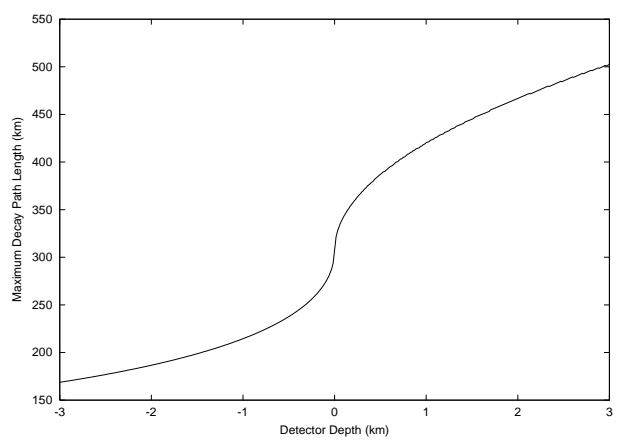

FIG. 2: The maximum decay path length as a function of detector depth. The curve is the maximum decay length relative to the maximum at zero detector depth. The depth axis runs from $3 \mathrm{~km}$ below to $3 \mathrm{~km}$ above the surface,
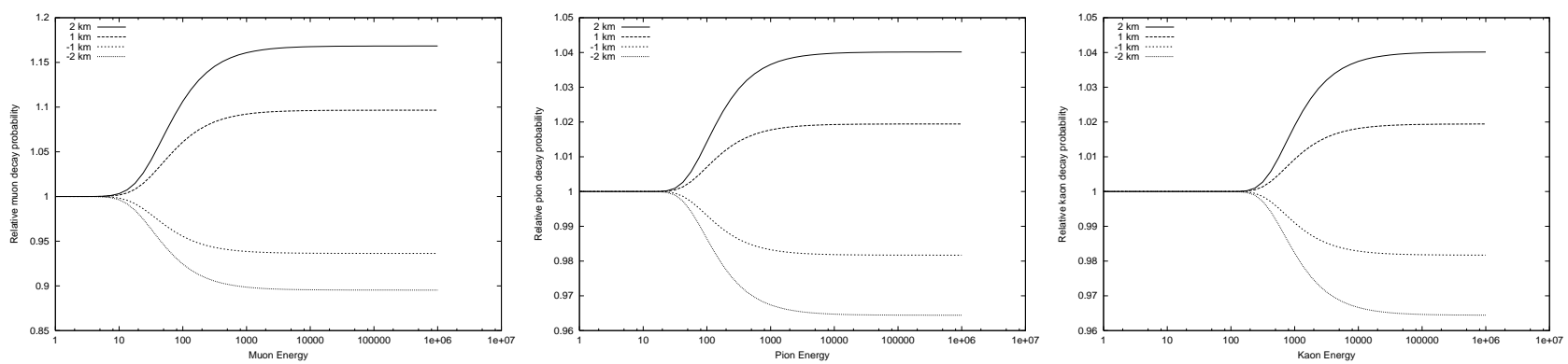

FIG. 3: The relative muon, pion and kaon decay probability as a function of energy. The four curves are for four different detector depths, $-2,-1,1$ and $2 \mathrm{~km}$. This is averaged over the zenith angle bin $-0.1<\cos \left(\theta_{z}\right)<0$.

\section{GEOMETRY}

Figure 1 illustrates the geometry. This figure is not to scale. We take $E$ to be the radius of the Earth, $6380 \mathrm{~km}$ and $A$ to be the radius at which neutrino source particles are produced. $B$ will be one interaction length below $A$. Most decays will occur between $A$ and $B$. $D$ represents the detector depth. If the detector is above sea level $D$ will be negative. We take as the decay length the difference in length for ray originating at $D$ and ending at a point along the ray at radius $A$ or $E$. It should be clear from figure 1 with its disproportionate scale that the decay length will depend on detector depth. For muons we take $B=E$, the surface of the Earth.

A particle initiating in the upper atmosphere will travel a distance $\mathrm{S}$ before being absorbed.

$$
S=\sqrt{A^{2}-\sin \theta_{z}^{2}(E-D)^{2}}-\sqrt{B^{2}-\sin \theta_{z}^{2}(E-D)^{2}}
$$

Very near the horizon, the particle path may not intersect $B$, if the detector is above sea level $\left(\sin \theta_{z}{ }^{2}(E-D)^{2}>B^{2}\right)$. In that case we approximate the decay length by the distance from the upper atmosphere to the detector.

$$
S=-(E-D) \cos \theta_{z}+\sqrt{A^{2}-\sin \theta_{z}^{2}(E-D)^{2}}
$$

$\theta_{z}$ is the zenith angle. Note for upward going neutrinos $\cos \theta_{z}<0$.

In figure 2 we illustrate this effect for muons, where we take $B=E$ and $A=E+7.4 \mathrm{~km}$. The figure shows the maximum decay length for muons below the horizon. In most cases this maximum is obtained at the horizon. But when the detector is above sea level, $D<0$, the maximum decay path length is achieved near the horizon. Notice that the decay path length increases with the detector height.

Common detector depths, $D$ are in the range of $-1 \mathrm{~km}<D<2 \mathrm{~km}$. Detectors located in the mountains tend to be deep underground but well above sea level.

\section{MAGNITUDE OF THE EFFECT}

In figure 3 we explore the relative flux, as a function of neutrino parent particle energy, for 4 different detector depths. This figure plots the contribution to the neutrino flux of a detector at $D=-2,-1,1$ or $2 \mathrm{~km}$. relative to the 

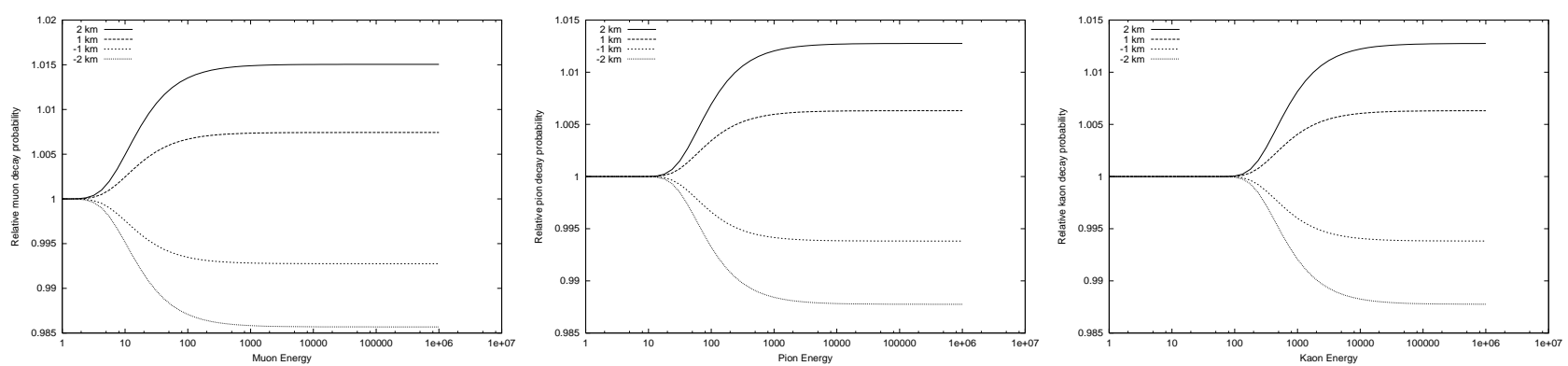

FIG. 4: The relative muon, pion and kaon decay probability as a function of energy. The four curves are for four different detector depths. This is averaged over the zenith angle bin $-0.2<\cos \left(\theta_{z}\right)<-0.1$
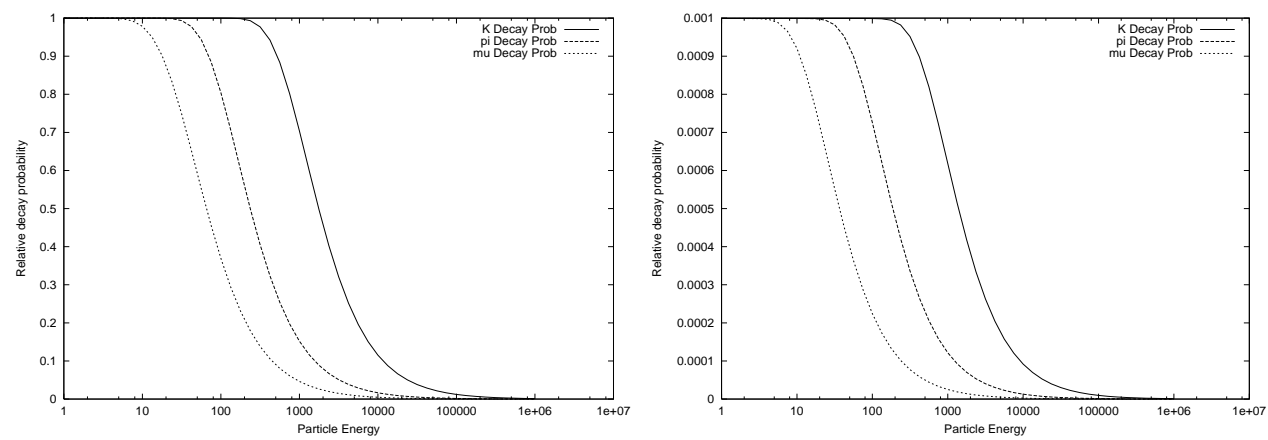

FIG. 5: The muon, pion and kaon decay probability as a function of energy for $D=0$. The one on the left is averaged over the zenith angle bin $-0.1<\cos \left(\theta_{z}\right)<0$. The one on the right is averaged over $-0.2<\cos \left(\theta_{z}\right)<-0.1$. The shorter path lengths in the $-0.2<\cos \left(\theta_{z}\right)<-0.1$ region yield a softer flux and lower event rate

contribution to the neutrino flux for a detector located at sea level $(D=0)$. The flux is averaged over the solid angle region of $-0.1<\cos \left(\theta_{z}\right)<0$., the angular bin just below the horizon. Variations are about $5 \%$ to $10 \%$.

In figure 3 one sees that the enhancement is not present at low energies, where all particles will decay. There is a transition region at moderate energies where the decay length is comparable to the absorption length. At the highest energies the decay probability scales linearly with the available decay length so the flux differences directly reflect the path length differences due to detector depth. The shape differences for muons, pions and kaons are due to the differences in masses, lifetimes and absorption lengths.

Figure 4 is similar to figure 3 except that now the solid angle region $-0.2<\cos \left(\theta_{z}\right)<-0.1$ is considered. Variations are now of the order of $0.6 \%$ to $1.2 \%$. The much lower flux modification away from the horizon indicates that standard neutrino flux calculations, that do not include the detector depth, will not correctly represent the angular distribution.

Figure 5 plots the muon, pion and kaon decay probability as a function of decay particle energy. The contribution at high energies is dominated by the particle with the shortest lifetime.

Figure [ 6 illustrates the depth effect in hadronic shower evolution. The multiple curves are for hadrons initiating at greater depth into the atmosphere. Each curve is 2 absorption lengths deeper in than the one to its right. The depth modulation is maintained at approximately the same amplitude, but as showers go deeper into the atmosphere the curves move to lower energies since the absorption length drops. Higher energy hadrons are more likely to be absorbed than to decay when the are formed lower in the atmosphere.

\section{LIMITATIONS}

This paper has made no attempt to quantitatively sum the various contributions to the atmospheric neutrino flux. Muons, pions and kaons all contribute but their relative contributions depend on their initial fractions and the fate of the other contributions. At very high energies "prompt" sources of neutrinos such as charm decay become important. At the highest energies muons produced via decays of the form $\rho \rightarrow \mu^{+} \mu^{-}$and $J / \psi \rightarrow \mu^{+} \mu^{-}$, or Drell-Yan processes constitute a significant source of neutrino flux. 


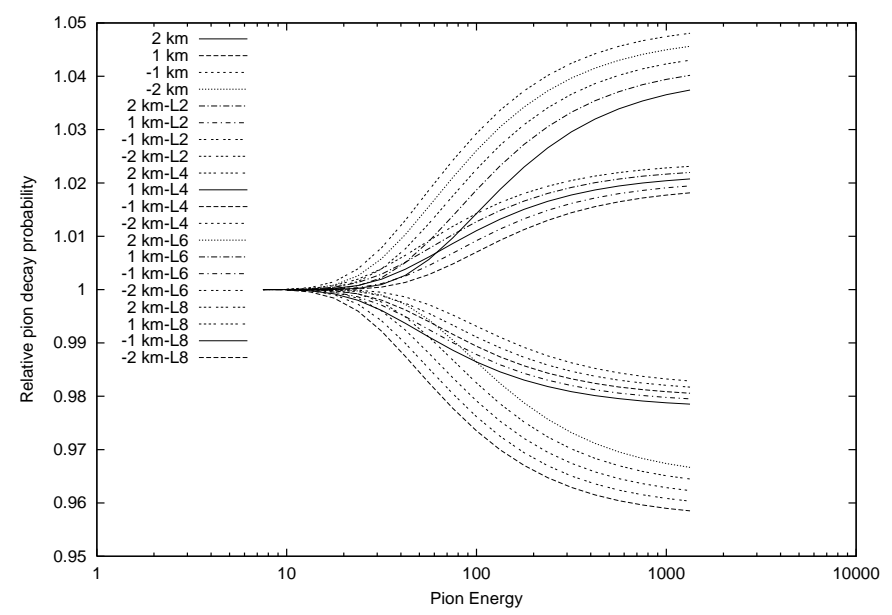

FIG. 6: Hadronic shower development brings the enhancement to lower energies. The 4 branches are the 4 detector depths considered in figures 3 and 4 The multiple curves in each branch are for hadrons initiating 2 absorption lengths lower in the atmosphere than for the curves to their right.

\section{CONCLUSIONS}

Our analysis suggests that there is a modest location dependent contribution to the high energy atmospheric neutrino flux. Detectors above sea level (but still underground) will see enhancements of the high energy flux in the vicinity of the horizon. This would be manifest as an angular distortion and an increased rate. Detectors below sea level would expect to see the opposite effect, with fewer high energy events and fewer events near the horizon. While these effects are of the order of $5 \%$ to $10 \%$ they contribute a systematic distortion when comparing data taken at different locations or when comparing observations from a deep detector to a flux estimate for a sea level location.

\section{ACKNOWLEDGMENTS}

Todor Stanev has recently reached similar conclusions about the influence of detector depth. I would like to thank O. Ryazhskaya for pointing out the significance of muon production via hadronic resonance decay $\left(\rho \rightarrow \mu^{+} \mu^{-}\right)$as a major source of neutrinos at high energies. This work was supported in part by the US Department of Energy under grant DE-FG02-00ER41145.

[1] M. Ambrosio et al., Phys. Lett. B434, 451 (1998).

[2] S. Hatakeyamma et al., Phys. Rev. Lett. 81, 2016 (1998).

[3] R. Becker-Szendy et al., Phys. Rev. Lett. 69, 1010 (1992).

[4] G.G. McGrath, "A Treatise on High Energy Muons in the IMB Detector", Ph.D. thesis, University of Hawaii at Manoa (1993).

[5] Y. Oyama, "Experimental Study of Upward-Going Muons in Kamiokande", Ph.D. thesis, Institute for Cosmic Ray Research, University of Tokyo (1989). 